required, with a focus on the size distribution of mineral grains, to attain a well-constrained assessment of the olivine composition ${ }^{7}$.

In future work, Li et al. should characterize at the landing site not only soil samples but also samples of rock. This task could be carried out through a comprehensive in situ exploration of the area that surrounds the Chang'e-4 landing site, with the acquisition of reflectance spectra from selected bedrock targets. Such exploration is also crucial to better document the geological context of the detected materials, so that potential issues that might call into question the authors' interpretation of their results can be addressed. These issues include the possibility that the impact that created the South Pole-Aitken Basin led to the formation of a massive sheet of melted rock that is tens of kilometres thick and has compositionally distinct layers ${ }^{8,9}$. Another complexity could arise from the regional emplacement, after the formation of the South Pole-Aitken Basin, of cryptomaria $^{10}$ (lava flows buried by subsequent crater ejecta).
Nevertheless, Li and colleagues' results are thrilling and could have considerable implications for characterizing the composition of the Moon's upper mantle ${ }^{11-13}$, and for establishing constraints on characteristics of the lunar magma ocean that would have varied with time. Such characteristics include the ocean's depth, its rate of cooling and its rate of evolution - the latter of which is controlled by magma viscosity, convection processes and the subsequent development of instability. In a broader sense, the authors' findings might also affect our understanding of the formation and evolution of planetary interiors. It is of the utmost importance to make progress towards unpacking the geology of the lunar far side, expanding our fundamental knowledge of the Moon's formation and the origin of the crustal asymmetry that exists between its near and far sides $^{14}$, and preparing future sample-return missions.

Patrick Pinet is at the Research Institute in Astrophysics and Planetology (IRAP), CNRS/
CNES/UPS/the University of Toulouse,

Toulouse 31400, France.

e-mail:patrick.pinet@irap.omp.eu

1. Li, C. et al. Nature 569, 378-382 (2019).

2. Wood, J. A., Dickey, J. S. Jr, Marvin, U. B. \& Powell, B. N. Geochim. Cosmochim. Acta 1, 965-988 (1970).

3. Elardo, S. M., Draper, D. S. and Shearer, C. K. Jr. Geochim. Cosmochim. Acta 75, 3024-3045 (2011).

4. Jolliff, B. L., Gillis, J. J., Haskin, L. A., Korotev, R. L. \& Wieczorek, M. A. J. Geophys. Res. Planet. 105, 4197-4216 (2000).

5. Wieczorek, M. A. et al. Rev. Mineral. Geochem. 60, 221-364 (2006).

6. Wieczorek, M. A. et al. Science 339, 671-675 (2013).

7. Clénet, H. et al. Icarus 213, 404-422 (2011).

8. Hurwitz, D. M. \& Kring, D. A. J. Geophys. Res. Planet. 119, 1110-1133 (2014).

9. Vaughan, W. M. \& Head, J. W. Planet. Space Sci. 91, 101-106 (2014).

10.Pieters, C. M., Head, J. W. III, Gaddis, L., Jolliff, B. \& Duke, M. J. Geophys. Res. Planet. 106, 2800128022 (2001).

11.Charlier, B., Grove, T. L., Namur, O. \& Holtz, F. Geochim. Cosmochim. Acta 234, 50-69 (2018).

12. Moriarty, D. P. III \& Pieters, C. M. J. Geophys. Res. Planet. 123, 729-747 (2018).

13.Melosh, H. J. et al. Geology 45, 1063-1066 (2017).

14. Ohtake, M. et al. Nature Geosci. 5, 384-388 (2012).

\title{
Scaling up quantum simulations
}

\section{It is difficult to carry out and verify digital quantum simulations that use many quantum bits. A hybrid device based on a digital classical computer and an analog quantum processor suggests a way forward. SEE ARTICLE P.355}

\section{JÜRGEN BERGES}

$\mathrm{D}$ evices known as universal quantum computers can be programmed to run different algorithms, thereby dispensing with the need to build new quantum computers for different functions. Fully fledged digital quantum simulations on such a device would allow substantial progress to be made in a wide range of disciplines, from quantum chemistry and materials science to fundamental high-energy physics. However, for this approach, it remains difficult to incorporate the many quantum bits (qubits) that are required for complex simulations. By contrast, large-scale analog quantum simulators already exist in today's laboratories. But, unlike their digital counterparts, these simulators are not programmable in general, and are often considered to be dedicated, single-purpose machines. Now, on page 355, Kokail et al. ${ }^{1}$ report a programmable analog quantum simulator that is versatile and has the potential to be scalable.

The authors' simulations run on a combination of a classical digital computer and an analog quantum processor. The latter does the

hard work of preparing trial states - quantum states that are used to evaluate physical quantities. The classical computer analyses the results of these evaluations, with the aim of optimizing certain adjustable (variational) parameters on which the trial states depend. This computer then suggests improved parameters to its quantum co-worker in a feedback loop. In

"There are many pressing problems

for which simulators involving analog quantum devices could be a gamechanger."

Kokail and colleagues use this set-up to carry out quantum simulations of the ground state of electrons coupled to light - a system that is described by the theory of quantum electrodynamics in one spatial dimension. Some of the authors were involved in the first digital simulation of this theory, on a four-qubit universal quantum computer ${ }^{2}$. By contrast, the current set-up includes up to 20 qubits. And although it is non-universal, the same analog quantum simulator could be used to study a wide range of models that can be defined on a lattice in condensed-matter and high-energy physics. These applications are restricted only by general requirements, such as the symmetries of the physical system to be simulated.

Similar hybrid classical-quantum algorithms have been demonstrated, using up to six qubits, in quantum chemistry, condensed-matter physics and high-energy physics ${ }^{3-5}$. With regard to the key property of potential scalability, the computational capabilities of the classical computer involved can be very restrictive. However, Kokail et al. show a promising way forward, which is mainly based on identifying and using approximate physical symmetries to reduce the number of variational parameters that need to be determined by the classical computer.

Kokail and colleagues also address the crucial question of how to verify that the outcome of a large-scale quantum simulation is correct. In addition to computing the mean energy of the ground state in their set-up, the authors calculate how much the energy varies from this mean value. They find that the variance is small enough for the results to be considered reliable. The ground-state energy of quantum electrodynamics might not seem the best example with which to demonstrate the power of the authors' approach - in principle, the results in this case could be obtained on any ordinary computer using standard classical-simulation techniques. But it is precisely this fact that makes the example a valuable test case.

There are many other pressing problems for which simulators involving analog quantum devices could be a game-changer. An 
example of such a problem is the Hubbard model ${ }^{6}$, which is a model of the dynamics of electrons arranged on a periodic lattice. Despite its simplicity and potential for use in a variety of technological applications, the Hubbard model remains a difficult problem in general. Electrons are fermions - particles whose key property is that only one of them can occupy a particular quantum state at any given time. Such behaviour is most efficiently simulated using an analog device, rather than a digital one. It is an exciting prospect for future applications that fermions could be incorporated into Kokail and colleagues' versatile set-up, with the help of a quantum processor containing ultracold fermionic atoms.

In general, large-scale analog quantum simulators could be much more versatile than anticipated on the basis of their behaviour with only a few qubits. This is because quantum many-body systems can behave very differently from few-body ones. For instance, in high-energy physics, the 'empty' vacuum is already a complex quantum system that requires a huge number of qubits to simulate it. However, only a small subset of the microscopic details of the underlying theory is relevant for the computation of measurable physical properties, using a concept known as the renormalization program of quantum field theory ${ }^{7}$. As a result, for an analog quantum simulation of such an underlying theory, many of the detailed properties of the device have no effect on the outcome of the simulation.

An extreme case is when the dynamics of a quantum many-body system becomes fully 'universal'. Here, universality means that certain measurable properties have no dependence on microscopic details. Then, a single analog quantum device could be used to simulate all physical systems that belong to the same universality class, subject only to general requirements such as physical symmetries. This concept is well established for some groundstate or static properties. But universality has now been extended to run analog quantum simulations of dynamical aspects far from the ground state, such as the dynamics of the early Universe, with the help of ultracold atoms ${ }^{8,9}$.

As Kokail and colleagues point out, future developments might bridge or even close some of the gaps between the concepts of analog and digital quantum simulation. The authors' work is a key step in that direction.

Jürgen Berges is at the Institute for Theoretical Physics, Heidelberg University, Heidelberg 69120, Germany.

e-mail:berges@thphys.uni-heidelberg.de

1. Kokail, C. et al. Nature 569, 355-360 (2019).

2. Martinez, E. A. et al. Nature 534, 516-519 (2016).

3. O'Malley, P. J. J. et al. Phys. Rev. X 6, 031007 (2016).

4. Kandala, A. et al. Nature 549, 242-246 (2017).

5. Klco, N. et al. Phys. Rev. A 98, 032331 (2018).

6. The Hubbard model at half a century. Nature Phys. 9, 523 (2013).

7. Weinberg, S. The Quantum Theory of Fields (Cambridge Univ. Press, 1995).

8. Prüfer, M. et al. Nature 563, 217-220 (2018)

9. Erne, S., Bücker, R., Gasenzer, T., Berges, J. \& Schmiedmayer, J. Nature 563, 225-229 (2018).

\section{Protein assembles into Archimedean geometry}

A natural protein has been engineered to self-associate into an architecture previously unknown among biological molecules: a cage structure based on one of the classic polyhedra identified by Archimedes. SEE LETTER P.439

\section{TODD O. YEATES}

A combination of ideas from geometry, computer science and molecular biology is ushering in a golden age for the design of nanometre-scale materials made from protein building blocks ${ }^{1}$. Naturally occurring cellular and viral structures highlight the diverse architectures that can be formed from protein molecules, and also hint at possible technological applications for designer proteins with predetermined shapes ${ }^{2}$. On page 439, Malay et al. ${ }^{3}$ report the production of a surprising and extraordinary protein structure: a cage-like architecture composed of 264 protein subunits held together at their edges by gold ions.

Efforts to make geometric protein architectures have generally focused on symmetrical 3D shapes. In particular, the Platonic solids ${ }^{4}-$ which include the tetrahedron, cube and icosahedron - have provided strategic design targets owing to their geometric simplicity ${ }^{5}$. These architectures can be realized by arrangements in which multiple protein subunits occupy identical spatial environments in the protein assembly.

Cubes, for example, have been constructed ${ }^{6-8}$ from protein trimers (complexes of three identical protein subunits). Each trimer occupies a corner of a cube, so that eight of them are needed to form the complete shape (Fig. 1a). Each subunit in a trimer can contact a subunit from another trimer along one of the edges of the cube. Those contacts are all identical, and hold the assembly together.

All three types of symmetry embodied by the Platonic solids - tetrahedral, octahedral and icosahedral - have been assembled using protein building blocks ${ }^{6-9}$. But the family of Platonic solids consists of just five shapes, which limits the architectures that can be made, and also constrains the geometries of the protein building blocks that can be used to construct them. This raises the question of what other shapes could be constructed using building blocks whose geometries make them unsuitable for making Platonic solids. A membrane-protein assembly described in 2014 showed evidence of unusual possibilities ${ }^{10}$.

Enter Malay et al., who started with a naturally occurring protein that forms a ringshaped assembly containing 11 identical copies of the protein molecule (an undecamer). They engineered the protein's sequence to incorporate a cysteine amino-acid residue, which has a thiol group ( $\mathrm{SH}$ ) in its side chain. The resulting undecamer ring therefore presents 11 thiol groups around its perimeter. Thiol groups can bind to metal atoms, so the perimeter thiol groups allow multiple undecamers to assemble into larger structures through thiol-metal interactions.

Malay and colleagues observed that the undecamers assembled into cage-like structures when mixed with a source of either gold or mercury ions. The authors knew that the 11-fold symmetry of the undecamers was incompatible with the construction of a Platonic solid, but the flexibility and reversibility of metal-mediated interactions could allow a diverse range of other architectures to form ${ }^{11}$. Characterizing the structure was challenging, but the researchers ultimately succeeded in visualizing it in atomic detail using cryo-electron microscopy.

The structure is of a type not seen before in molecular systems: 24 copies of the 11-membered ring are held together by specific interactions between the rings. Malay et al. identified the arrangement as a snub cube, which belongs to a group of polyhedra known as Archimedean solids. ${ }^{4}$ The vertices in Archimedean solids are all equivalent, but the faces and edges can be of distinct types.

Each vertex in the snub cube is connected by the edges to five other vertices, but - in contrast to the case for Platonic solids - the angles between the connections at a given vertex are not all the same. Instead, one of the angles is larger than the other four (Fig. 1b). Fortuitously for Malay et al., the edges of the snub cube very nearly match up with the alternating 'spokes' that radiate from the centre of an undecagon to its edges. This means that one of the authors' undecamers can be placed at each of the 24 vertices of a snub cube to make 